\title{
Total synthesis of solamargine
}

\author{
Guohua Wei ${ }^{\mathrm{a}}$, Jing Wang ${ }^{\mathrm{a}}$, Yuguo Du ${ }^{\mathrm{a}, \mathrm{b}, *}$ \\ a State Key Laboratory of Environmental Chemistry and Ecotoxicology, Research Center for Eco-Environmental Sciences (CAS), Beijing 100085, China \\ ${ }^{\mathrm{b}}$ College of Chemistry and Chemical Engineering, Graduate University of Chinese Academy of Sciences, Beijing 100049, China
}

\section{A R T I C L E I N F O}

\section{Article history:}

Received 22 November 2010

Revised 25 February 2011

Accepted 17 March 2011

Available online 23 March 2011

\section{Keywords:}

Solasodine

Natural products

Solamargine

Cytotoxic activity

Glycosides

\begin{abstract}
A B S T R A C T
Solamargine, $(25 R)-3 \beta-\{0-\alpha-\mathrm{L}-$ rhamnopyranosyl-( $1 \rightarrow 2)-[0-\alpha$-L-rhamnopyranosyl-( $1 \rightarrow 4)]-\beta$-D-glucopyranosyloxy\}-22 $\alpha-N$-spirosol-5-ene, has been synthesized in 13 steps in a $10.5 \%$ overall yield starting from the naturally abundant diosgenin. Condensation of a partially protected glucopyranosyl donor with an oxaza-spiro moiety, which was formed in one-pot azido reduction, significantly improved the synthesis of desired molecule. The target compound exhibited good cytotoxic activities against tumor cells HeLa, A549, MCF-7, K562, HCT116, U87, and HepG2 with IC 50 ranging from 2.1 to $8.0 \mu \mathrm{M}$.
\end{abstract}

(c) 2011 Elsevier Ltd. All rights reserved.
Solamargine is a major glycoalkaloid which occurs in at least 100 Solanum species. ${ }^{1}$ Pharmacological studies have indicated that solamargine and its derivatives show anti-tumor activities through inhibition of tumor cell growth, such as colon (HT-29, HCT-15), prostate (LNCap, PC-3), breast (T47D, MDA-MB-231), and human hepatoma (PLC/PRF/5) cells. ${ }^{2}$ Despite its widespread occurrence and biological importance, there are surprisingly few reports of the total synthesis of solamargine or structurally related compounds. ${ }^{3}$ Solamargine is structurally composed of two parts: an oxaza-spiro steroidal aglycone (solasodine) and a chacotriose (2,4-bis- $\alpha$-L-rhamnopyranosyl- $\beta$-D-glucopyranose) attached to the 3-hydroxy group of solasodine. We have previously described an efficient method for the preparation of natural saponins possessing 2,4-branched oligosaccharides using partially protected glycosyl donors ${ }^{4}$ and would like to report here an extension of this strategy to a facile total synthesis of natural solamargine and its anti-tumor activities.

We envisaged that solamargine $(\mathbf{1})$ could be constructed from a fully acetylated L-rhamnopyranosyl bromide $\mathbf{2}$ and a well-defined glycoside $\mathbf{3}$. Compound $\mathbf{3}$ could be prepared through a coupling reaction between solasodine $\mathbf{4}$ and a partially protected thioglucopyranoside 5. In designing this glucosyl donor, PMB ( $p$-methoxybenzyl) was selected as the protecting groups, as it not only enhances the reactivity of the sugar moiety, but it also improves the selectivity in the final deprotection step (Fig. 1). This building block could also facilitate the continuous $\mathrm{C}-2^{\prime}$ and $\mathrm{C}-4^{\prime}$ glycosylation due to the accessible free 2,4-hydroxyl groups. Solasidine 4

\footnotetext{
* Corresponding author. Tel./fax: +86 1062849126.

E-mail address: duyuguo@rcees.ac.cn (Y. Du).
}

would be synthesized using a one-pot reductive ring closure from the known compound $\mathbf{6}$.

The synthesis of $\mathbf{4}$ started with the known compound $\mathbf{6}$ which was obtained from the commercially available diosgenin in three steps and a $50 \%$ overall yield. ${ }^{5}$ Treatment of $\mathbf{6}$ with $\mathrm{K}_{2} \mathrm{CO}_{3}$ in $\mathrm{MeOH}$ then generated spiroketal $\mathbf{7}$ and its counterpart dione $\mathbf{8}$. These two compounds were found to be interconvertible in organic solution and consequently, the mixture of $\mathbf{7}$ and $\mathbf{8}$ was treated directly with p-toluenesulfonyl chloride/pyridine $(\rightarrow \mathbf{9})$, followed by azido-substitution with $\mathrm{NaN}_{3}$, to give $\mathbf{1 0}$ in $85 \%$ yield over three steps. It has been well documented that selective reduction of the 16-ketone of the cholestan-16,22-dione with $\mathrm{NaBH}_{4}$ in $i$-PrOH provided the corresponding furostan through a concurrent intramolecular hemiketal formation. ${ }^{6}$ Applying the same idea, dione $\mathbf{1 0}$ was successfully converted into the hemiketal $\mathbf{1 1}$ in a moderate yield of $62 \%$. The key reductive-cyclization of compound $\mathbf{1 1}$ was carried out smoothly in the presence of $\mathrm{Ph}_{3} \mathrm{P}$ under refluxing conditions, and followed by desilylation with $6 \mathrm{~N} \mathrm{HCl}$ in one-pot, furnished solasodine 4 in $95 \%$ yield over two steps (Scheme 1). The stereochemistry on C-22 is fully controlled as the free amine attacks C-22 from the less hindered back face, and the structure of 4 was confirmed by comparison of its physical data with those reported for the natural solasodine. ${ }^{7,8,14}$

The partially protected thioglycoside donor $\mathbf{5}$ was prepared from compound $\mathbf{1 2}^{4}$ and $p$-methoxybenzylidenation of 12 with anisaldehyde dimethyl acetal $(\rightarrow \mathbf{1 3})$, followed by tin-assisted regioselective $p$-methoxybenzylation, ${ }^{9}$ provided 14 in a yield of $70 \%$ from 12. Selective ring opening ${ }^{10}$ of $\mathbf{1 4}$ with sodium cyanoborohydride and trifluoroacetic acid proceeded smoothly to afford $\mathbf{5}$ in $85 \%$ yield (Scheme 2 ). 


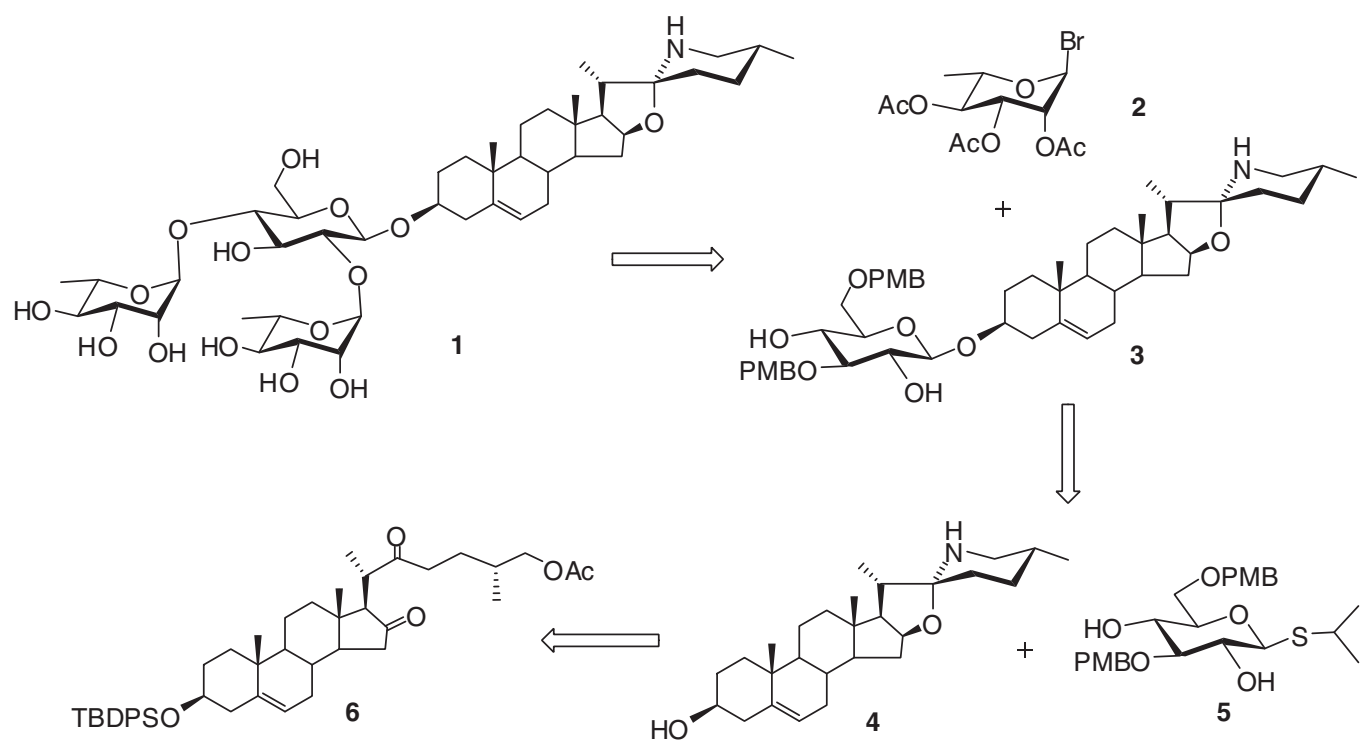

Figure 1. Retrosynthetic analysis of solamargine $\mathbf{1 .}$

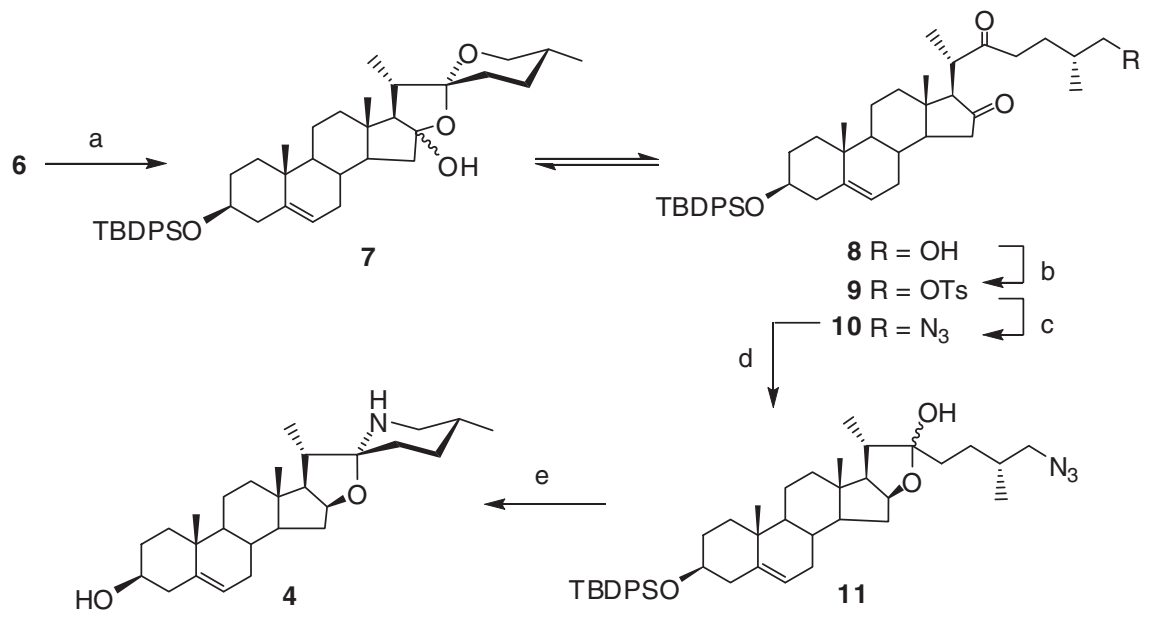

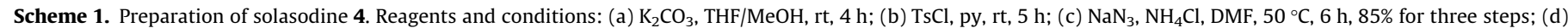
$\mathrm{NaBH}_{4}, i-\mathrm{PrOH}, \mathrm{rt}, 5 \mathrm{~h}, 62 \%$; (e) $\mathrm{Ph}_{3} \mathrm{P}, \mathrm{THF} / \mathrm{H}_{2} \mathrm{O}$, reflux, 2 h; $6 \mathrm{~N} \mathrm{HCl}, \mathrm{EtOH}$, reflux, 2 h, 95\% for two steps.

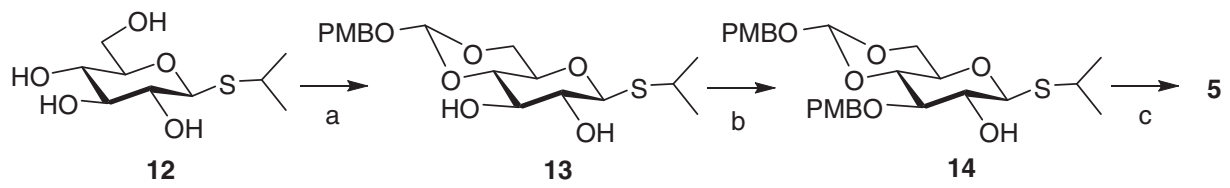

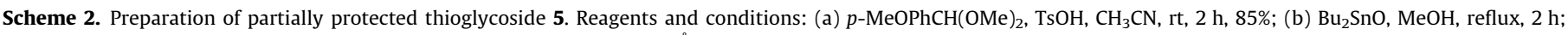
$\mathrm{PMBCl}, \mathrm{Bu}_{4} \mathrm{NI}$, toluene, $80^{\circ} \mathrm{C}, 6 \mathrm{~h}, 82 \%$ for two steps; (c) $\mathrm{NaCNBH}_{3}$, TFA, $4 \AA \mathrm{MS}, \mathrm{DMF}, 0{ }^{\circ} \mathrm{C}, 6 \mathrm{~h}, 85 \%$.

With building blocks 4 and $\mathbf{5}$ in hand, the efficient synthesis of solamargine $\mathbf{1}$ was initiated. Coupling of solasodine $\mathbf{4}$ and a partially protected glycosyl donor $\mathbf{5}$ was carried out in dry methylene dichloride in the presence of AgOTf and $\mathrm{N}$-iodosuccinimide (NIS) at $-50{ }^{\circ} \mathrm{C}$ generating the desired key saponin 3 in $65 \%$ isolated yield after a preparative HPLC purification. ${ }^{11}$ In this glycosylation, AgOTf was found to be a more effective promoter than the more commonly used TMSOTf (trimethylsilyl trifluoromethanesulfonate), probably because of the existence of the secondary amine in 4 . A doublet at $4.36 \mathrm{ppm}(\mathrm{J}=7.8 \mathrm{~Hz})$ in ${ }^{1} \mathrm{H}$ NMR spectrum clearly indicated the $\beta$-configuration of $\mathbf{3}$. Condensation of $\mathbf{3}$ and L-rhamnopyranosyl bromide $2^{12}$ in the presence of AgOTf at $-10^{\circ} \mathrm{C}$ generated saponin 15 in a yield of $81 \%$. Finally, removal of the PMB groups from compound 15 using $10 \% \mathrm{TFA}^{13}$ in $\mathrm{CH}_{2} \mathrm{Cl}_{2}$ at $-15^{\circ} \mathrm{C}$, followed by global deacylation with $0.3 \mathrm{M} \mathrm{NaOH}$ in $\mathrm{MeOH}$ afforded the target compound $\mathbf{1}$ in $80 \%$ yield over two steps (Scheme 3). Remarkably, this complex natural saponin was prepared convergently in 13 steps and in a $10.5 \%$ overall yield. The analytic data ${ }^{14}\left({ }^{13} \mathrm{C}\right.$ NMR, MS, optical rotation) for the synthetic solamargine $\mathbf{1}$ were identical to those reported for the natural product. ${ }^{1}$

The cytotoxic activities of synthetic solamargine 1 on tumor cells HeLa, A549, MCF-7, K562, HCT116, U87, and HepG2, as well as two normal cell lines (HL7702 and H9C2), were evaluated fol- 


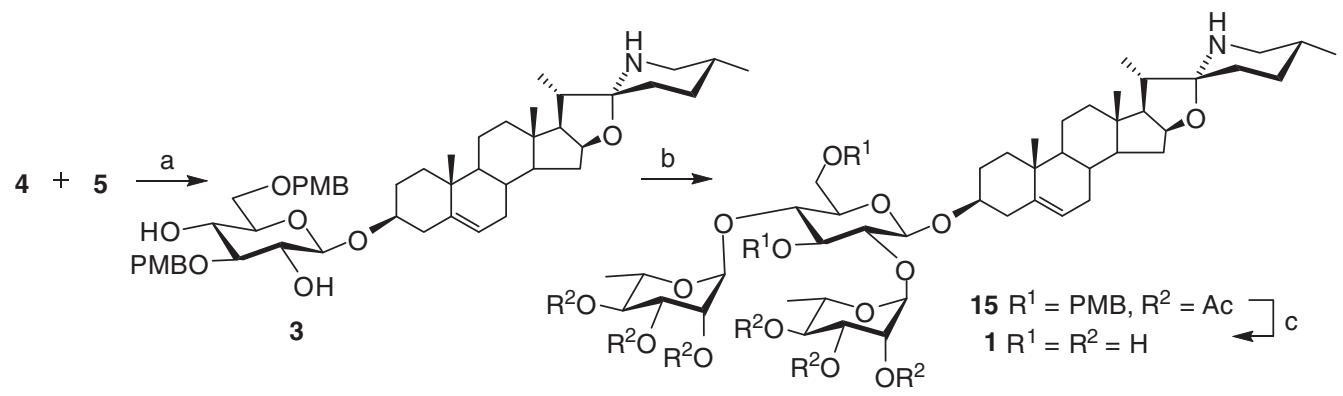

Scheme 3. Synthesis of solamargine 1. Reagents and conditions: (a) NIS, AgOTf, $\mathrm{CH}_{2} \mathrm{Cl}_{2},-50{ }^{\circ} \mathrm{C}, 2 \mathrm{~h}, 65 \%$; (b) $2, \mathrm{AgOTf} \mathrm{CH}_{2} \mathrm{Cl}_{2},-10{ }^{\circ} \mathrm{C}, 2 \mathrm{~h}, 81 \%$; (c) $10 \% \mathrm{TFA}^{2} \mathrm{Cn} \mathrm{CH}_{2} \mathrm{Cl} 2,-15{ }^{\circ} \mathrm{C}$, $1 \mathrm{~h} ; 0.3 \mathrm{M} \mathrm{NaOH}, \mathrm{MeOH}, \mathrm{rt}, 4 \mathrm{~h}, 80 \%$ for two steps.

Table 1

Cytotoxicity of compound $\mathbf{1}$ on seven tumor cell lines $\left(\mathrm{IC}_{50}, \mu \mathrm{M}\right)^{\mathrm{a}}$

\begin{tabular}{llllllll}
\hline & HeLa & A549 & MCF-7 & K562 & HCT116 & U87 & HepG2 \\
\hline $\mathbf{1}$ & 6.0 & 8.0 & 2.1 & 5.2 & 3.8 & 3.2 & 2.5 \\
Cisplatin & 26.3 & $>30$ & 17.0 & 23.1 & 18.5 & $>30$ & 9.8 \\
\hline
\end{tabular}

${ }^{\text {a }}$ Values are means of three independent experiments.

Table 2

Cytotoxicity of compounds $\mathbf{1}$ on two normal cell lines ( $\left.\mathrm{IC}_{50}, \mu \mathrm{M}\right)^{\mathrm{a}}$

\begin{tabular}{lll}
\hline & HL7702 & H9C2 \\
\hline $\mathbf{1}$ & 13.5 & $>20$ \\
\hline
\end{tabular}

${ }^{a}$ Values are means of three independent experiments.

lowing the standard MTT assay. ${ }^{15}$ Tables 1 and 2 show the inhibition of tumor cell growth by solamargine $\mathbf{1}$ with $\mathrm{IC}_{50}$ ranging from 2.1 to $8.0 \mu \mathrm{M}$, but exhibited a lower cytotoxicity to the normal hepatocyte cell HL7702.

In conclusion, the natural product solamargine has been chemically synthesized in 13 steps in a 10.5\% overall yield starting from the natural abundant diosgenin. Application of one-pot reductive-cyclization to form the spirosolan derivative followed by condensation with a partially protected glucopyranosyl donor has significantly simplified the saponin synthesis. The approach described here should be valuable for the related molecule ${ }^{16}$ design, synthesis, and bioactivity screening.

\section{Acknowledgments}

This work was supported in partial by NNSF of China (projects 30701043, 20732001, 20890112) and project 2009ZX09501-011. We would like to thank Dr. Vicky Gibson of Carbosynth for helping with the manuscript preparation and Dr. Jianjun Zhang of CAU for providing compounds $\mathbf{2}$ and $\mathbf{1 2}$ as the starting materials.

\section{References and notes}

1. Wanyonyi, A. W.; Chhabra, S. C.; Mkoji, Gerald; Eilert, Udo; Njue, W. M. Phytochemistry 2002, 59, 79.

2. (a) Kuo, K. W.; Hsu, S. H.; Li, Y. P.; Lin, W. L.; Liu, L. F.; Chang, L. C.; Lin, C. C.; Lin, C. N.; Sheu, H. M. Biochem. Pharmacol. 2000, 60, 1865; (b) Liu, L. F.; Liang, C. H.; Shiu, L. Y.; Lin, W. L.; Lin, C. C.; Kuo, K. W. FEBS Lett. 2004, 577, 67; (c) Al Chami, L.; Méndez, R.; Chataing, B.; O'Callaghan, J.; Usubillaga, A.; LaCruz, L. Phytother. Res. 2003, 17, 254; (d) Maruo, V. M.; Soares, M. R.; Bernardi, M. M.; Spinosa, H. S. Neurotoxicol. Teratol. 2003, 25, 627; (e) Heo, K. S.; Lee, S. J.; Ko, J. H.; Lim, K.; Lim, K. T. Toxicol. In Vitro 2004, 18, 755.

3. Shahid, M. PCT Patent WO/018604, 2003.

4. Du, Y.; Gu, G.; Wei, G.; Hua, Y.; Linhardt, R. J. Org. Lett. 2003, 5, 3627.

5. (a) Cheng, M. S.; Wang, Q. L.; Tian, Q.; Song, H. Y.; Liu, Y. X.; Li, Q.; Xu, X.; Miao, H. D.; Yao, X. S.; Yang, Z. J. Org. Chem. 2003, 68, 3658; (b) Yu, B.; Liao, J. C.; Zhang, J. B.; Hui, Y. Z. Tetrahedron Lett. 2001, 42, 77.
6. Mazur, Y.; Danieli, N.; Sondheimer, F. J. Am. Chem. Soc. 1960, 82, 5889.

7. Bird, G. J.; Collins, D. J.; Eastwood, F. W.; Exter, R. H.; Romanelli, M. L.; Small, D. D. Aust. J. Chem. 1979, 32, 783.

8. Puri, R.; Wong, T. C.; Puri, R. K. Magn. Reson. Chem. 1993, 31, 278.

9. Nashed, M. A.; Anderson, L. Tetrahedron Lett. 1976, 17, 3530.

10. Garegg, P. J. Pure Appl. Chem. 1984, 56, 849.

11. A general procedure for the glycosylation was described as follows: Silver triflate (AgOTf, 0.3 equiv of $\mathbf{5}$ ) was added to a solution of $\mathrm{N}$-iodosuccinimide (NIS, 1.5 equiv of $\mathbf{5})$ in a mixture of $\mathrm{CH}_{2} \mathrm{Cl}_{2} /$ toluene $(10: 1, \mathrm{v} / \mathrm{v})$. To a mixture of $\mathbf{5}$ (1.2 equiv of 4 ) and 4 in dry $\mathrm{CH}_{2} \mathrm{Cl}_{2}$ at $-50{ }^{\circ} \mathrm{C}$ under nitrogen was added dropwise the above solution of NIS/AgOTf and the progress was monitored by TLC analysis. After complete disappearance of the starting materials, the reaction was quenched by adding a $10 \%$ aq $\mathrm{Na}_{2} \mathrm{~S}_{2} \mathrm{O}_{3}$ solution. The organic layer was washed with $10 \%$ aq $\mathrm{Na}_{2} \mathrm{~S}_{2} \mathrm{O}_{3}$ solution, dried over $\mathrm{Na}_{2} \mathrm{SO}_{4}$, and concentrated in vacuo. The residue was purified by preparative HPLC to obtain the pure product.

12. Hansen, T.; Daasbjerg, K.; Skrydstrup, T. Tetrahedron Lett. 2000, 41, 8645.

13. Zhan, Z. Y.; Ollmann, I. R.; Ye, X. S.; Wischnat, R.; Baasov, T.; Wong, C. H. J. Am. Chem. Soc. 1999, 121, 734.

14. The selected physical data. Compound 4: $[\alpha]_{\mathrm{D}}^{25}-112\left(c^{2} 0.81, \mathrm{CHCl}_{3}\right)$ [lit. ${ }^{7}$ $\left.[\alpha]_{\mathrm{D}}^{26}-116.7\left(\mathrm{c} 0.62, \mathrm{CHCl}_{3}\right)\right]$. Selected ${ }^{1} \mathrm{H}$ NMR $\left(400 \mathrm{MHz}, \mathrm{CDCl}_{3}\right): \delta 0.82(\mathrm{~s}, 3 \mathrm{H}$, $\mathrm{H}-18), 0.84$ (d, 3H, J = 6.2 Hz, H-27), 0.96 (d, 3H, J = 7.2 Hz, H-21), 1.03 (s, 3H, H19), 2.20-2.33 (m, 2H, H-4a, H-4b), 2.58-2.68 (m, 2H, H-26a, H-26b), 3.48-3.55 $(\mathrm{m}, 1 \mathrm{H}, \mathrm{H}-3 \alpha), 4.28-4.30(\mathrm{~m}, 1 \mathrm{H}), 5.35(\mathrm{~d}, 1 \mathrm{H}, \mathrm{J}=5.1 \mathrm{~Hz}, \mathrm{H}-6)$; selected ${ }^{13} \mathrm{C}$ NMR $\left(100 \mathrm{MHz}, \mathrm{CDCl}_{3}\right): \delta 15.08,16.22,19.07,19.27,20.86,30.16,31.46,31.66$, $32.02,32.11,32.12,33.99,36.62,37.24,39.90,40.52,41.30,42.30,47.63,50.19$, $56.52,62.91,71.65,78.98,98.14,121.24,140.84$. ESI-HRMS calcd for $\mathrm{C}_{27} \mathrm{H}_{43} \mathrm{NO}_{2}$ : $413.3294[\mathrm{M}]^{+}$, found: $414.3364[\mathrm{M}+\mathrm{H}]^{+}$. Compound 3: $[\alpha]_{\mathrm{D}}^{25}-43$ (c $\left.0.55, \mathrm{CHCl}_{3}\right)$; selected ${ }^{1} \mathrm{H}$ NMR $\left(400 \mathrm{MHz}, \mathrm{CDCl}_{3}, 25^{\circ} \mathrm{C}\right): \delta 0.81(\mathrm{~s}, 3 \mathrm{H}, \mathrm{H}-18)$, 0.86 (d, 3H, $J=6.2 \mathrm{~Hz}, \mathrm{H}-27$ ), 0.96 (d, 3H, J = 7.2 Hz, H-21), 1.03 (s, 3H, H-19), 2.60-2.66 (m, 2H, H-26a, H-26b), 3.36-3.49 (m, 3H), 3.55-3.60 (m, 2H), 3.66 $\left(\mathrm{dd}, 1 \mathrm{H}, J=5.4,10 \mathrm{~Hz}, \mathrm{H}-6 \mathrm{a}^{\mathrm{I}}\right), 3.72\left(\mathrm{dd}, 1 \mathrm{H}, J=5.4,10 \mathrm{~Hz}, \mathrm{H}-6 \mathrm{~b}^{\mathrm{I}}\right), 3.80(\mathrm{~s}, 6 \mathrm{H}$, $\left.\mathrm{CH}_{3} \mathrm{OPhCH}_{2}\right), 4.32(\mathrm{~m}, 1 \mathrm{H}, \mathrm{H}-16), 4.36\left(\mathrm{~d}, 1 \mathrm{H}, J_{1,2}{ }^{\mathrm{I}}=7.8 \mathrm{~Hz}, \mathrm{H}-1^{\mathrm{I}}\right), 4.50(\mathrm{~s}, 2 \mathrm{H}$, $\left.\mathrm{CH}_{3} \mathrm{OPhCH}\right)_{2}, 4.72\left(\mathrm{~d}, 1 \mathrm{H}, J=11 \mathrm{~Hz}\right.$, one proton of $\left.\mathrm{CH}_{3} \mathrm{OPhCH}_{2}\right), 4.88(\mathrm{~d}, 1 \mathrm{H}$, $J=11 \mathrm{~Hz}$, one proton of $\left.\mathrm{CH}_{3} \mathrm{OPhCH}_{2}\right), 5.34(\mathrm{~d}, 1 \mathrm{H}, J=5.0 \mathrm{~Hz}, \mathrm{H}-6), 6.85-7.32(\mathrm{~m}$, $8 \mathrm{H}, \mathrm{Ph})$; selected ${ }^{13} \mathrm{C}$ NMR $\left(100 \mathrm{MHz}, \mathrm{CDCl}_{3}\right): \delta 15.35,16.36,19.19,19.39$, $20.87,29.68,29.71,31.20,31.43,31.76,32.09,32.15,36.87,37.26,38.90,39.80$ $40.62,42.38,47.67,50.10,55.28$ (2 C), 56.45, 62.94, 70.30, 71.81, 73.29, 74.10 74.13, 74.26, 79.04, 83.42 (2 C), 98.40, 101.26, 121.79, 140.40, 159.31, 159.39. ESI-HRMS calcd for $\mathrm{C}_{49} \mathrm{H}_{69} \mathrm{NO}_{9}: 815.4972[\mathrm{M}]^{+}$, found: $816.5042[\mathrm{M}+\mathrm{H}]^{+}$. Compound 15: $[\alpha]_{\mathrm{D}}^{25}+68\left(\right.$ c $\left.0.6, \mathrm{CHCl}_{3}\right)$; selected ${ }^{1} \mathrm{H} \mathrm{NMR}\left(400 \mathrm{MHz}, \mathrm{CDCl}_{3}\right): \delta$ 0.81 (s, 3H, H-18), 0.88 (d, 3H, $J=6.2 \mathrm{~Hz}, \mathrm{H}-27), 1.01$ (m, 6H, H-21, 19), 1.19$1.26\left(\mathrm{~m}, 6 \mathrm{H}, \mathrm{H}-6^{\mathrm{II}}, 6^{\mathrm{III}}\right), 1.71,1.72,1.87,2.01,2.03,2.05\left(6 \mathrm{~s}, 6 \times 3 \mathrm{H}, \mathrm{CH}_{3} \mathrm{CO}\right)$, 2.62-2.68 (m, 2H, H-26a, H-26b), 3.36-3.57 (m, 7H), 3.67-3.72 (m, 2H), $3.78(2$ $\left.\mathrm{s}, 2 \times 3 \mathrm{H}, \mathrm{CH}_{3} \mathrm{OPhCH}_{2}\right), 4.32-4.34(\mathrm{~m}, 1 \mathrm{H}, \mathrm{H}-16), 4.39\left(\mathrm{~d}, 2 \mathrm{H}, J_{1.2} \mathrm{II}=7.8 \mathrm{~Hz}, \mathrm{H}-\right.$ $\left.1^{\mathrm{I}}\right), 4.48\left(\mathrm{~s}, 2 \mathrm{H}, \mathrm{CH}_{3} \mathrm{OPhCH}\right), 4.57\left(\mathrm{~d}, 1 \mathrm{H}, J=10.0 \mathrm{~Hz}\right.$, one proton of $\left.\mathrm{CH}_{3} \mathrm{OPhCH}_{2}\right)$, $4.63\left(\mathrm{dd}, 1 \mathrm{H}, J=2.6,4.0 \mathrm{~Hz}, \mathrm{H}-2^{\mathrm{II}}\right), 4.68\left(\mathrm{dd}, 1 \mathrm{H}, J=2.6,4.0 \mathrm{~Hz}, \mathrm{H}-2^{\mathrm{III}}\right), 4.74(\mathrm{~d}$, $1 \mathrm{H}, J=10.0 \mathrm{~Hz}$, one proton of $\left.\mathrm{CH}_{3} \mathrm{OPhCH}\right), 4.98-5.04\left(\mathrm{~m}, 3 \mathrm{H}, \mathrm{H}-3^{\mathrm{III}}, 4^{\mathrm{II}}, 4^{\mathrm{III}}\right)$, $5.10\left(\mathrm{dd}, 1 \mathrm{H}, J=4.0,8.6 \mathrm{~Hz}, \mathrm{H}-3^{\mathrm{II}}\right), 5.19\left(\mathrm{~d}, 1 \mathrm{H}, J=2.0 \mathrm{~Hz}, \mathrm{H}-1^{\mathrm{II}}\right), 5.23(\mathrm{~d}, 1 \mathrm{H}$, $\left.J=2.0 \mathrm{~Hz}, \mathrm{H}-1^{\mathrm{III}}\right), 5.34(\mathrm{~d}, 1 \mathrm{H}, J=5.0 \mathrm{~Hz}, \mathrm{H}-6), 6.85-7.32(\mathrm{~m}, 8 \mathrm{H}, P h) .{ }^{13} \mathrm{C}$ NMR $\left(100 \mathrm{MHz}, \mathrm{CDCl}_{3}\right) \delta 15.3,16.4,17.5,17.7,19.3,19.4,20.5,20.7,20.8,20.9,22.7$ 25.7, 26.1, 29.7 (2 C), 29.9, 31.4, 31.9, 32.1, 32.2, 36.9, 37.3, 38.6, 39.9, 40.6, 41.3, 47.5, 50.1, 55.3 (2 C), 56.5, 62.5, 69.2, 69.3, 69.5, 70.1, 70.3, 70.4, 70.8, 72.3, $72.9(2 \mathrm{C}), 74.6,74.9,75.0,76.0,79.0,81.9(2 \mathrm{C}), 97.1\left(\mathrm{C}-1^{\mathrm{II}}\right), 97.4\left(\mathrm{C}-1^{\mathrm{III}}\right)$, 98.3 (C-22), $100.7\left(\mathrm{C}-1^{\mathrm{I}}\right), 121.7(\mathrm{C}-6), 140.7(\mathrm{C}-5), 159.1(2 \mathrm{C}), 169.7\left(2 \mathrm{CH}_{3} \mathrm{CO}\right)$, $169.8\left(2 \mathrm{CH}_{3} \mathrm{CO}\right), 170.2\left(\mathrm{CH}_{3} \mathrm{CO}\right), 170.3\left(\mathrm{CH}_{3} \mathrm{CO}\right)$. ESI-HRMS calcd for $\mathrm{C}_{73} \mathrm{H}_{101} \mathrm{NO}_{23}$ : $1359.6764[\mathrm{M}]^{+}$, found: $1360.6833[\mathrm{M}+\mathrm{H}]^{+}$. Compound 1: $\left.[\alpha]_{\mathrm{D}}^{25}-88(c) 0.5, \mathrm{MeOH} / \mathrm{CHCl}_{3}=1: 1\right) \quad\left[\begin{array}{ll}\text { lit. } & 1\end{array}[\alpha]_{\mathrm{D}}^{24}-91\right.$ (c $0.2, \quad \mathrm{MeOH} /$ $\left.\left.\mathrm{CHCl}_{3}=1: 1\right)\right]$. Selected ${ }^{1} \mathrm{H}$ NMR $\left(400 \mathrm{MHz}, \mathrm{CDCl}_{3} / \mathrm{CD}_{3} \mathrm{OD}=1: 1\right): \delta 0.84(\mathrm{~s}, 3 \mathrm{H}$, $\mathrm{H}-18), 0.87(\mathrm{~d}, 3 \mathrm{H}, J=6.2 \mathrm{~Hz}, \mathrm{H}-27), 0.97(\mathrm{~d}, 3 \mathrm{H}, J=7.0 \mathrm{~Hz}, \mathrm{H}-21), 1.05(\mathrm{~s}, 3 \mathrm{H}, \mathrm{H}-$ 19), 1.22-1.26 (m, 6H, H-6 $\left.6^{\mathrm{II}}, 6^{\mathrm{III}}\right), 1.42-2.47$ (m, 24H), 2.63-2.67 (m, 2H, H-26), $3.21-4.09(\mathrm{~m}, 15 \mathrm{H}), 4.31-4.33(\mathrm{~m}, 1 \mathrm{H}, \mathrm{H}-16), 4.50\left(\mathrm{~d}, 1 \mathrm{H}, J=7.8 \mathrm{~Hz}, \mathrm{H}-1^{\mathrm{I}}\right), 4.90$ $\left(\mathrm{s}, 1 \mathrm{H}, \mathrm{H}-1^{\mathrm{II}}\right), 5.24\left(\mathrm{~s}, 1 \mathrm{H}, \mathrm{H}-1^{\mathrm{III}}\right), 5.40(\mathrm{~d}, 1 \mathrm{H}, J=4.0 \mathrm{~Hz}, \mathrm{H}-6)$. Selected ${ }^{13} \mathrm{C}$ NMR $\left(100 \mathrm{MHz}, \mathrm{CDCl}_{3}: \mathrm{CD}_{3} \mathrm{OD}=1: 1\right): \delta 15.6,16.9,17.4,17.9,19.2(2 \mathrm{C}), 20.8,28.4$, 28.7, 29.2, 29.6, 30.2, 31.5, 33.4, 36.2, 38.2, 39.1, 40.5, 41.5, 42.1, 47.3, 51.1, $56.0,61.4,62.8,68.2,68.9,69.2,69.4,70.5,71.5,73.3,73.5,75.5,76.8,77.1$, 
79.1, 79.5, 79.6, 98.8, 100.4, 101.3, 102.4, 121.8, 141.3. ESI-HRMS calcd for $\mathrm{C}_{45} \mathrm{H}_{73} \mathrm{NO}_{15}: 867.4980[\mathrm{M}]^{+}$, found: $868.5051[\mathrm{M}+\mathrm{H}]^{+}$.

15. Kuroda, M.; Mimaki, Y.; Sashida, Y.; Hirano, T.; Oka, K.; Dobashi, A.; Li, H.; Harada, N. Tetrahedron 1997, 53, 11549.

16. (a) Blankemeyer, J. T.; McWilliam, M. L.; Rayburn, J. R.; Weissenberg, M.; Friedman, M. Food Chem. Toxicol. 1998, 36, 383; (b) Gao, J.; Li, X.; Gu, G.; Sun, B.;
Cui, M.; Ji, M.; Lou, H.-X. Bioorg. Med. Chem. Lett. 2011, 21, 622; (c) Yang, S.-W.; Chan, T.-M.; Buevich, A.; Priestley, T.; Crona, J.; Reed, J; Wright, A. E.; Patel, M.; Gullo, V.; Chen, G.; Pramanik, B.; Chu, M. Bioorg. Med. Chem. Lett. 2007, 17, 5543; (d) Gauthier, C.; Legault, J.; Piochon, M.; Lavoie, S.; Tremblay, S.; Pichette, A. Bioorg. Med. Chem. Lett. 2009, 19, 2310. 